\title{
Revising Darcy's law: \\ a necessary step toward progress in fluid mechanics and reservoir engineering
}

\author{
C. Ketata, M. G. Satish \& M. R. Islam \\ Department of Civil Engineering, Dalhousie University, Canada
}

\begin{abstract}
After drilling wells to reach an oil and gas reservoir, its production starts following the fluid flow under surrounding pressure. To characterize an oil and gas reservoir and estimate its production correctly, it is paramount to model its fluid mechanics properly. So far, the main models used to simulate oil and gas flow utilize Darcy's law. However, these run short due to its limited applications and lack of adaptability in oil and gas reservoirs. This paper introduces a novel fluid transport law in porous media that can be used in oil and gas reservoir, as well as in civil, chemical, mechanical, and mineral engineering cases. This comprehensive model describes the oil and gas flow in a reservoir efficiently. It proposes that the pressure gradients in the flow directions depend not only on the fluid velocity but also on a power series and a series of first and higher order partial derivatives of fluid velocities, among other factors. The coefficients in these series are specific to the fluids and rocks representing the reservoir. They portray the fluid-rock interaction. They include rock properties such as composition, porosity, and permeability. Porosity is the ratio of the space taken up by the pores in a rock to its total volume. The pore space determines the amount of space available for storage of fluids. Permeability is the ability of a rock to allow fluids to pass through it. In addition, the flow model is affected by fluid types and properties such as composition, density, and viscosity. Viscosity is the property of a fluid that causes it to resist flowing.

Keywords: fluid flow in porous media, Darcy's law, fluid mechanics, oil and gas engineering, reservoir engineering.
\end{abstract}




\section{Introduction}

Fluid mechanics is the branch of mechanics that deals with the properties of gases and liquids, their motion, and their application in practical engineering such as oil and gas engineering dealing with oil and gas reservoirs. An oil and gas reservoir is a natural chamber in a porous rock where a supply of natural gas and crude oil collects. To solve oil and gas reservoir engineering problems, it is essential to observe, model, simulate, and understand fluid flow motion in porous media. Darcy's law has been used for a long time to describe water and other fluids flow through porous media $[1,2]$.

Darcy [1] derived an equation that governs the laminar or nonturbulent flow of fluids in homogeneous porous media. In 1855 and 1856, he conducted column experiments that established Darcy's law for flow in sands and therefore the theoretical foundation of groundwater hydrology [1]. Darcy's law is a phenomenological law rather than a fundamental law [3]. Various researchers [4-18] in fluid mechanics and oil and gas engineering accommodated Darcy's law to meet their needs in order to solve complex problems dealing with fluid flow in porous media including oil and gas flow in reservoir rock layers.

To curb the limitations of Darcy's law to comparatively small fluid velocities, Forchheimer's equation and its inertial flow parameter, $\beta$, have been used as an extension of Darcy's law beyond the linear flow region $[4,17]$. According to Darcy [1], the concept of a constant permeability suggests a linear relationship between flowrate and pressure gradient. However, empirical observations of flowrate at higher differential pressures corroborated that the relationship between flowrate and pressure gradient becomes nonlinear at relatively high velocities [4, 17]. Forchheimer [4] presented an empirical equation that described the observed nonlinear flow behavior. Nevertheless, this new equation has showed limits too $[4,17]$. Consequently, it is necessary to develop a fundamental law by revealing a new model.

This paper introduces a novel model of power and partial derivative series describing the fluid flow motion in porous media and considering its dynamic spatial behavior.

\section{Darcy's law}

Darcy's law [1] expresses fluid motion in porous media. Darcy [1] conducted experiments on water flow through sand beds. He found out that the water velocity in the sand beds was proportional to the pressure gradient as follows:

$$
v=k \frac{h_{1}-h_{2}}{L}
$$

where $v$ is the water velocity, $k$ the hydraulic conductivity, $h_{i}$ the hydraulic head, and $\mathrm{L}$ the column length. The water velocity equals the water flowrate divided by the sand beds cross area:

$$
v=\frac{q}{A}
$$


Darcy's law is linear and applies only for incompressible and singlephase fluids following laminar and steady-state flows in homogeneous and isotropic porous media. However, the fluid flow can be laminar or turbulent depending on the porous media composition, porosity, and permeability, among others. In addition, other factors such as distance from the oil and gas wellbore, fluid composition, viscosity, and velocity determine the fluid flow conditions whether they are laminar or turbulent. The fluid flow can follow a steady-state, a pseudosteady state, or an unsteady-state regime. Moreover, Darcy's law is not a function of the flow direction. Therefore, it is obviously necessary to take into account the flow geometry to correct this flow motion law.

\section{Reservoir rock permeability}

Permeability is the ability of a rock to transmit fluids. It is influenced by rock properties such as its type, composition, porosity (see Figure 1), and grain shape and size, among others. Furthermore, fluid properties such as its type, composition, and viscosity establish its motion behavior in porous media. Viscosity is the property of a fluid that causes it to resist flowing. Based on lab flow tests, Darcy [1] determined that permeability could be expressed as follows (see Figure 2):

$$
k=Q \mu / A(\Delta P / L)
$$

where $k$ is the permeability (darcy), $Q$ the volumetric flowrate $\left(\mathrm{cm}^{3} / \mathrm{s}\right), \mu$ the viscosity of flowing medium (cp), $A$ the cross-section of porous medium such as rock $\left(\mathrm{cm}^{2}\right), \Delta P$ the pressure differential or drop, and $\mathrm{L}$ the porous medium length. Figure 3 shows how horizontal and vertical permeabilities are affected by rock grains arrangement, size, shape, and pore structure.

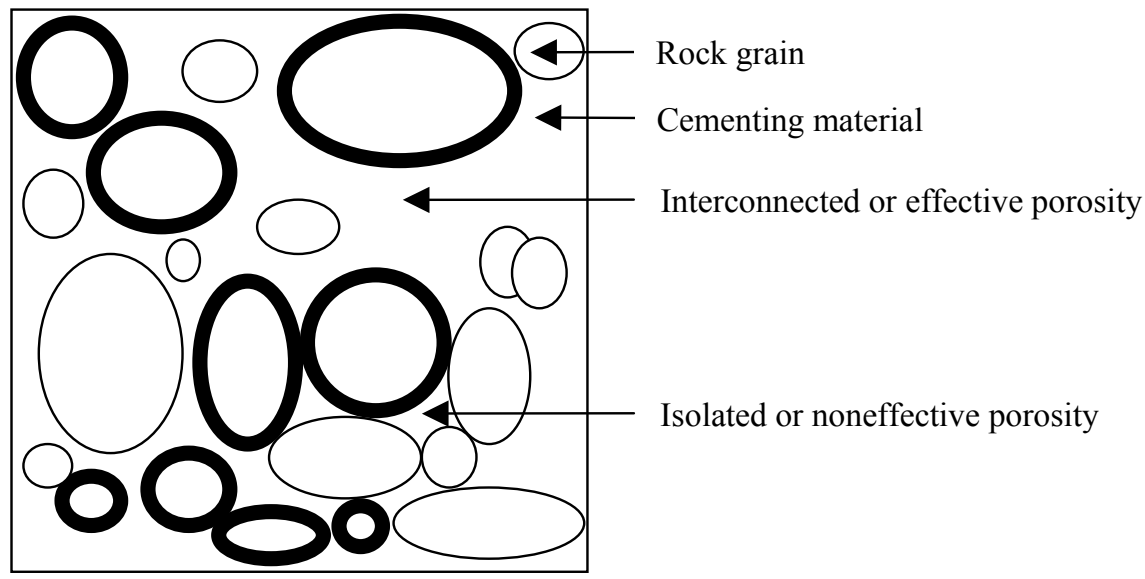

Figure 1: Rock porosity. 


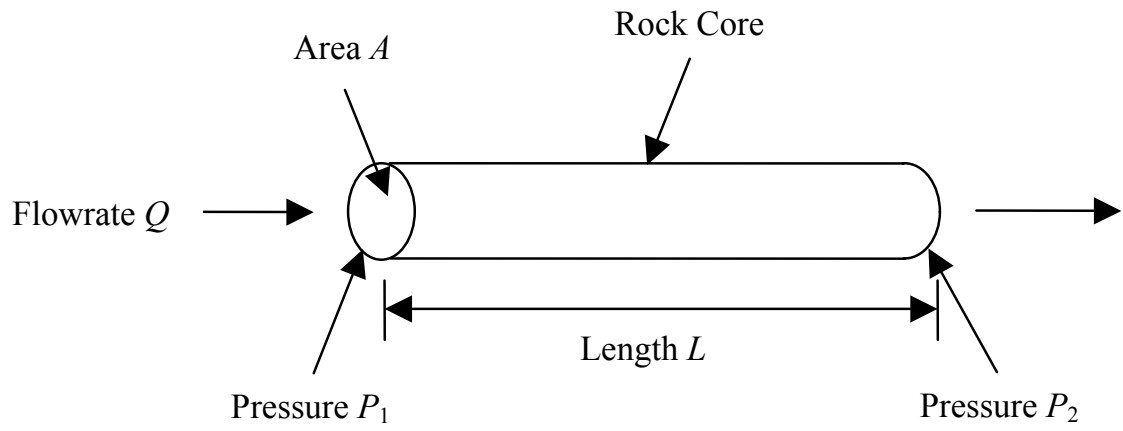

Figure 2: $\quad$ Rock core for permeability assessment.

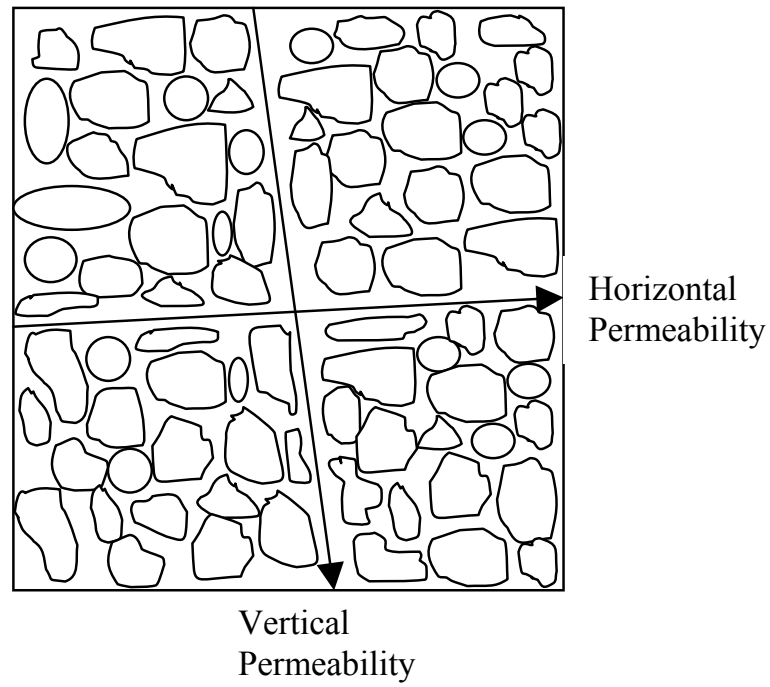

Figure 3: Horizontal and vertical permeabilities.

\section{Fluid types}

A fluid is a substance whose molecules flow freely, so it has no fixed shape and little resistance to outside stress. Fluids can be liquids or gases. Fluids can be slightly or considerably compressible (see Figures 4 and 5), whereas incompressible fluids do not exist.

\section{Flow regimes}

The fluid flow can be semisteady-state or unsteady-state as shown in Figure 6. However, since the pressure changes over time, a fluid flow cannot be steady- 
state. Furthermore, the fluid flow can be laminar or turbulent. At relatively low fluid velocities, the fluid particles adhere to the streamlines, which results in a laminar flow. At comparatively higher velocities, the fluid flow follows a fluctuating velocity pattern leading to a turbulent flow.

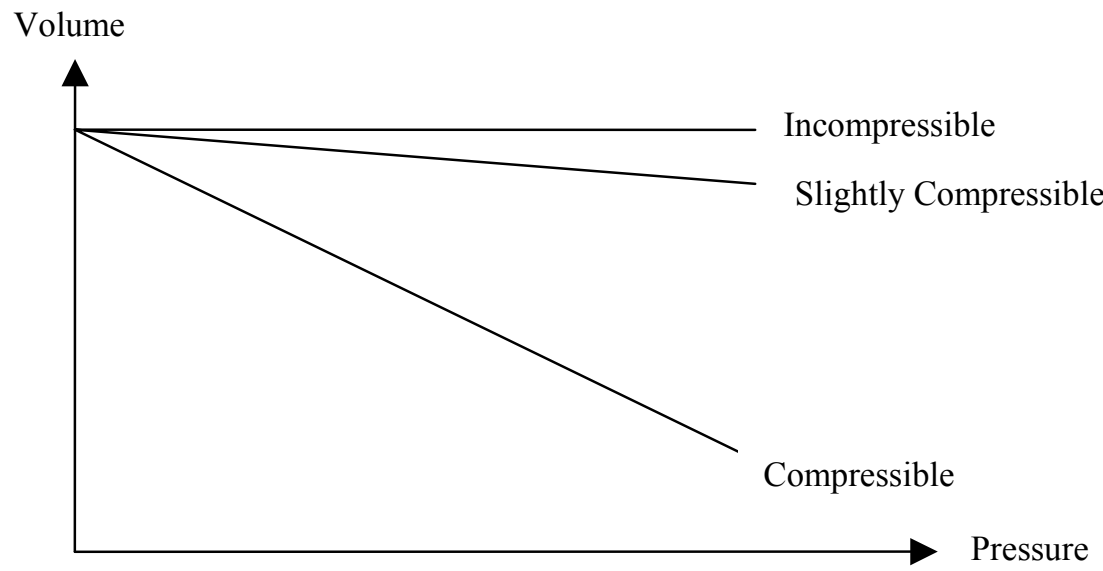

Figure 4: $\quad$ Fluid types depending on volume-pressure relationships.

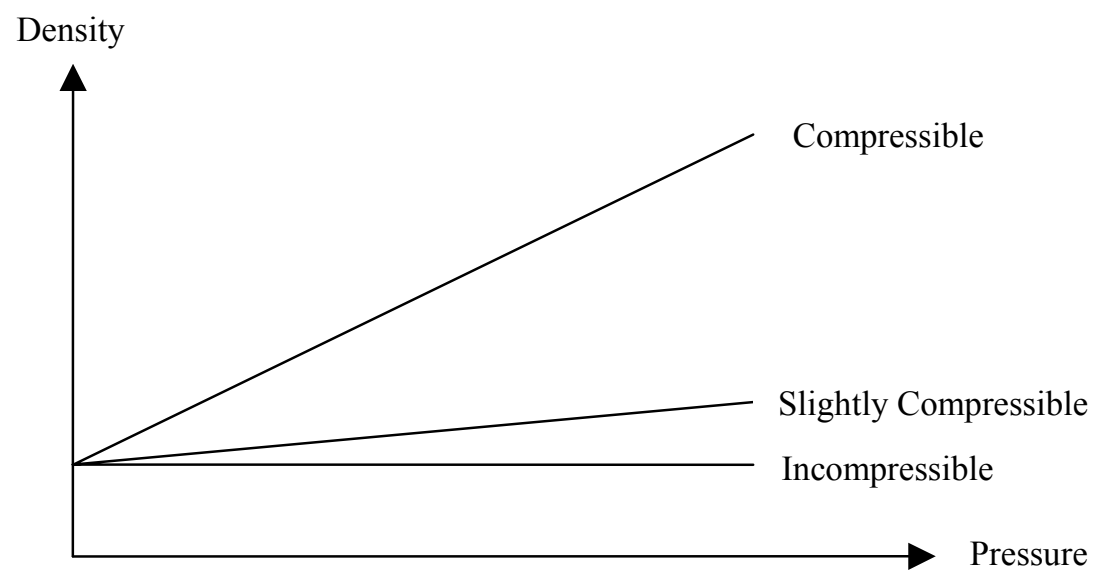

Figure 5: $\quad$ Fluid types depending on density-pressure relationships. 


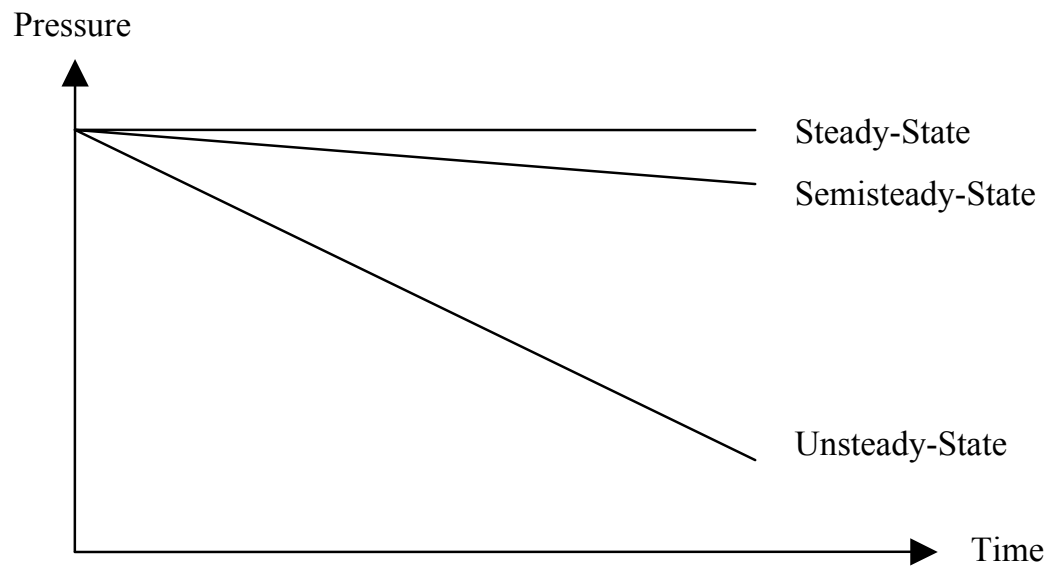

Figure 6: Fluid regimes depending on pressure-time relationships at a reservoir location.

\section{Power and Partial Derivative Series (PPDS) model}

Fluid flow in porous media is a nonlinear and dynamic process. The pressure differential at time $t$ is a function of fluid velocity variations as follows:

$$
\frac{\partial P}{\partial x}=\sum_{i=1}^{m} a_{i} v^{i}+\sum_{j=1}^{n} b_{j} \frac{\partial^{j} v}{\partial x^{j}}
$$

where the values of $m, a_{i}, n$, and $b_{j}$ depend on the porous medium properties and the fluid characteristics. Every lab or field case of fluid flow motion in porous media is unique. Therefore, these values are case-specific. After observing the flow behavior, the $\operatorname{PPDS}(m, n)$ model is calibrated. Next, the defined model is used for further site investigation and case simulations in order to understand, control, and forecast the dynamic flow system.

\section{Conclusion}

Darcy's law works only for incompressible and singlephase fluids following laminar and steady-state flows in homogeneous and isotropic porous media, which do not exist. Then, it is paramount to develop a new law that takes into account the attributes of a real fluid flow in porous media.

This paper proposes an innovative model for fluid flow motion in porous media considering the porous medium and fluid properties and parameters. This original model corresponds to a power series and a series of first and higher order partial derivatives of fluid velocities at the time of evaluation, which obeys the flow dynamic nonlinear behavior. 
Based on lab experiments and field data, the flow model is regulated since every case studied has a unique model. This novel and robust model not only embodies the fluid behavior in an oil and gas reservoir but also enhances the real-time decision making process for its production, which increases the project profitability.

\section{Acknowledgement}

The authors would like to thank the Atlantic Canada Opportunities Agency (ACOA) for funding this project under the Atlantic Innovation Fund (AIF).

\section{References}

[1] Darcy, H.P.G, The Public Fountains of the City of Dijon (Appendix), Bookseller of the Imperial Corps of Bridges, Highways and Mines, Quay of Augustins, 49, 1856, biosystems.okstate.edu/darcy.

[2] Ahmed, T., Reservoir Engineering Handbook, Second Edition, Butterworth-Heinemann: Woburn, 2001.

[3] Hofmann, J.R. \& Hofmann, P.A., Darcy's law and structural explanation in Hydrology. Proceedings of the 1992 Biennial Meeting of the Philosophy of Science Association, volume 1, eds. D. Hull, M. Forbes \& K. Okruhlik, Philosophy of Science Association: East Lansing, 1992.

[4] Forchheimer, P., Wasserbewegung durch Boden. Zeitschrift des Vereines Deutscher Ingenieure, 45, pp. 1782-1788, 1901.

[5] Firoozabadi, A. \& Katz, D.L., An analysis of high-velocity gas flow through porous media. Journal of Petroleum Technology, February, pp. 211-216, 1979.

[6] Thiruvengadam, M. \& Pradip Kumar, G.N., Validity of Forchheimer equation in radial flow through coarse granular media. Journal of Engineering Mechanics, 123(7), pp. 696-705, 1997.

[7] Andrade, J.S., Costa, U.M., Almeida, M.P., Makse, H.A. \& Stanley, H.E., Inertial effects on fluid flow through disordered porous media. Physical Review Letters, 82(26), pp. 5249-5252, 1999.

[8] Chen, Z., Qin, G. \& Ewing, R.E., Analysis of a compositional model for fluid flow in porous media. SIAM Journal on Applied Mathematics, 60(3), pp. 747-777, 2000.

[9] Rose, W., Myths about later-day extensions of Darcy's law. Journal of Petroleum Science and Engineering, 26, pp. 187-198, 2000.

[10] Saghir, M.Z., Chaalal, O. \& Islam, M.R., Numerical and experimental modeling of viscous fingering during liquid-liquid miscible displacement. Journal of Petroleum Science and Engineering, 26, pp. 253-262, 2000.

[11] Layton, W.J., Schieweck, F. \& Yotov, I., Coupling fluid flow with porous media flow. SIAM Journal on Numerical Analysis, 40(6), pp. 2195-2218, 2003.

[12] Belhaj, H.A., Agha, K.R., Nouri, A.M., Butt, S.D., Vaziri, H.H. \& Islam, M.R., Numerical simulation of non-Darcy flow utilizing the new 
Forchheimer's diffusivity equation. Paper 81499 presented at the SPE $13^{\text {th }}$ Middle East Oil Show and Conference in Bahrain, April 5-8, 2003.

[13] Belhaj, H.A., Agha, K.R., Nouri, A.M., Butt, S.D., Vaziri, H.H. \& Islam, M.R., Numerical modeling of Forchheimer's equation to describe Darcy and non-Darcy flow in porous media. Paper 80440 presented at the SPE Asia Pacific Oil and Gas Conference and Exhibition in Jakarta, Indonesia, April 15-17, 2003.

[14] Belhaj, H.A., Agha, K.R., Nouri, A.M., Butt, S.D. \& Islam, M.R., Numerical and experimental modeling of non-Darcy flow in porous media. Paper 81037 presented at the SPE Latin American and Caribbean Petroleum Engineering Conference and Exhibition in Port-of-Spain, Trinidad, West Indies, April 27-30, 2003.

[15] Belhaj, H.A., Agha, K.R., Butt, S.D. \& Islam, M.R., A comprehensive numerical simulation model for non-Darcy flow including viscous, inertial and convective contributions. Paper 85678 presented at the $27^{\text {th }}$ Annual SPE International Technical Conference and Exhibition in Abuja, Nigeria, August 4-6, 2003.

[16] Belhaj, H.A., Agha, K.R., Butt, S.D. \& Islam, M.R., Simulation of nonDarcy flow in porous media including viscous, inertial and frictional effects. Paper 84879 presented at the SPE International Improved Oil Recovery Conference in Asia Pacific in Kuala Lumpur, Malaysia, October 20-21, 2003.

[17] Barree, R.D. \& Conway, M.W., Beyond beta factors: A complete model for Darcy, Forchheimer, and trans-Forchheimer flow in porous media. Paper SPE 89325 presented at the SPE Annual Technical Conference and Exhibition in Houston, Texas, September 26-29, 2004.

[18] Miskimins, J.L., Lopez-Hernandez, H.D. \& Barree, R.D., Non-Darcy flow in hydraulic fractures: Does it really matter? Paper SPE 96389 presented at the SPE Annual Technical Conference and Exhibition in Dallas, Texas, October 9-12, 2005. 